\title{
Modeling of an EDLC with Fractional Transfer Functions Using Mittag-Leffler Equations
}

\author{
J. J. Quintana, ${ }^{1}$ A. Ramos, ${ }^{2}$ and I. Nuez ${ }^{1}$ \\ ${ }^{1}$ Electronic and Automatic Engineering Department, University of Las Palmas de G.C., 35017 Las Palmas, Spain \\ ${ }^{2}$ Process Engineering Department, University of Las Palmas de G.C., 35017 Las Palmas, Spain \\ Correspondence should be addressed to J. J. Quintana; jjquintana@diea.ulpgc.es
}

Received 31 July 2013; Revised 30 October 2013; Accepted 31 October 2013

Academic Editor: Bashir Ahmad

Copyright (C) 2013 J. J. Quintana et al. This is an open access article distributed under the Creative Commons Attribution License, which permits unrestricted use, distribution, and reproduction in any medium, provided the original work is properly cited.

\begin{abstract}
Electrochemical double-layer capacitors (EDLC), also known as supercapacitors or ultracapacitors, are devices in which diffusion phenomena play an important role. For this reason, their modeling using integer-order differential equations does not yield satisfactory results. The higher the temporal intervals are, the more problems and errors there will be when using integer-order differential equations. In this paper, a simple model of a real capacitor formed by an ideal capacitor and two parasitic resistors, one in series and the second in parallel, is used. The proposed model is based on the ideal capacitor, adding a fractional behavior to its capacity. The transfer function obtained is simple but contains elements in fractional derivatives, which makes its resolution in the time domain difficult. The temporal response has been obtained through the Mittag-Leffler equations being adapted to any EDLC input signal. Different charge and discharge signals have been tested on the EDLC allowing modeling of this device in the charge, rest, and discharge stages. The obtained parameters are few but identify with high precision the charge, rest, and discharge processes in these devices.
\end{abstract}

\section{Introduction}

In recent years, the growing demand for new electrical energy storing systems has led to a remarkable development of electrochemical double-layer capacitors (EDLC). The EDLC are devices capable of storing energy and are characterized by their very rapid response during charge and discharge cycles, which allows them to provide high power and to hold a high number of charge and discharge cycles.

The complementary qualities of EDLC and batteries have allowed the generation of numerous hybrid applications for energy recovery or storage systems. Energy storage in EDLC is not supported by chemical processes. Moreover, supercapacitors have a high life cycle and do not need any maintenance. From the above it can be concluded that because of their characteristics these devices have raised great interesting expectations $[1,2]$.

It is a usual procedure that the dynamics of real systems are modeled by differential equations. In most cases, the differential equations are based on conventional derivatives, yielding sufficiently accurate mathematical models. However, there are a variety of systems or phenomena in which mathematical models based on ordinary differential equations do not provide satisfactory solutions. One of these elements is the EDLC. It is this case, when the application of models based on fractional derivatives, studied in the field of fractional calculus, is very useful. These equations are called fractional differential equations.

In multiple fields of physics, there are numerous examples in which a differential equation is used as fractional modeling tool [3-5]. This is very common when considering geometry conditions with fractal dimension and distributed parameter systems [6-8]. In the field of electric power, the current through a capacitor is proportional to the noninteger-order integral for electric current $[9,10]$. An electric network composed of infinite RC elements can be modeled through fractional differential equations $[11,12]$.

In this paper, a dynamic fractional model for EDLC is developed, based on a differential equation with fractional 
derivatives. This equation has been solved by means of the Laplace transform, and in order to obtain the time domain solution used the Mittag-Leffler function $E_{\alpha, \beta}(z)$. The solution has been obtained for a unitary step input and for a generic input. This model has been applied to an EDLC for its identification in long time periods, obtaining very satisfactory results.

This paper is organized as follows: Section 2 shows a brief review of fractional calculus; in Section 3 the fractional model of the EDLC is deduced; Section 4 expounds the experimental data and the discussion; finally in Section 5 the conclusions are presented.

\section{Brief Review of Fractional Calculus}

In this section is exposed a brief mathematical background of the fractional calculus [3], for understanding methods, results, and conclusions presented in this paper. The following are some of the most popular functions, definitions, and properties of the fractional calculus.

2.1. Riemann-Liouville Derivative Definition. Riemann-Liouville definition expresses the fractional derivative as a time convolution integral

$$
\begin{array}{r}
{ }_{a} D_{t}^{\alpha} f(t)=\frac{1}{\Gamma(n-\alpha)} \frac{d^{n}}{d t^{n}} \int_{a}^{t} \frac{f(\tau)}{(t-\tau)^{\alpha-n+1}} d \tau \\
(n-1<\alpha<n),
\end{array}
$$

where $n$ is an integer and $\alpha$ is a real number and the fractional derivative order.

2.2. Caputo Derivative Definition. Caputo definition of a fractional derivative of a function is

$$
\begin{array}{r}
{ }_{a}^{C} D_{t}^{\alpha} f(t)=\frac{1}{\Gamma(n-\alpha)} \int_{a}^{t} \frac{f^{(n)}(\tau)}{(t-\tau)^{\alpha+1-n}} d \tau \\
(n-1 \leq \alpha<n),
\end{array}
$$

where $n$ is an integer and $\alpha$ is a real number and the fractional derivative order.

2.3. Grunwald-Letnikov Derivative Definition. GrunwaldLetnikov definition is a numerical form of the fractional derivative

$$
{ }_{a} D_{t}^{\alpha} f(t)=\lim _{h \rightarrow 0} \frac{1}{h^{\alpha}} \sum_{j=0}^{[(t-a) / h]}(-1)^{j}\left(\begin{array}{c}
\alpha \\
j
\end{array}\right) f(t-j h) .
$$

2.4. Laplace Transform of the Fractional Derivative. An interesting property of the fractional derivative operator is its Laplace transform

$$
\begin{aligned}
L\left[{ }_{0} D_{t}^{\alpha} f(t)\right]= & \int_{0}^{\infty} e^{-s t}{ }_{a} D_{t}^{\alpha} f(t) d t \\
= & s^{\alpha} F(s)-\left.\sum_{k=0}^{n-1} s^{k}{ }_{a} D_{t}^{\alpha-k-1} f(t)\right|_{t=0} \\
& \quad(n-1<\alpha \leq n),
\end{aligned}
$$

where, if initial conditions are null, it yields

$$
L\left[{ }_{0} D_{t}^{\alpha} f(t)\right]=s^{\alpha} F(s) .
$$

2.5. Mittag-Leffler Function. The two-parameter function of the Mittag-Leffler type $E_{\alpha, \beta}(z)$ plays a very important role in the fractional calculus and is defined as

$$
E_{\alpha, \beta}(z)=\sum_{k=0}^{\infty} \frac{z^{k}}{\Gamma(\alpha k+\beta)} \quad(\alpha>0, \beta>0) .
$$

The exponential function $e^{z}$ is a particular case of the MittagLeffler function

$$
E_{1,1}(z)=\sum_{k=0}^{\infty} \frac{z^{k}}{\Gamma(k+1)}=e^{z} .
$$

One of the main uses of the Mittag-Leffler function comes from the following Laplace transform

$$
\begin{aligned}
L\left[t^{\alpha k+\beta-1} E_{\alpha, \beta}^{(k)}\left( \pm a t^{\alpha}\right)\right] & =\int_{0}^{\infty} e^{-s t} t^{\alpha k+\beta-1} E_{\alpha, \beta}^{(k)}\left( \pm a t^{\alpha}\right) d t \\
& =\frac{k ! s^{\alpha-\beta}}{\left(s^{\alpha} \mp a\right)^{k+1}}
\end{aligned}
$$

where $k$ indicates the order of the derivative of the MittagLeffler function

$$
E_{\alpha, \beta}^{(k)}(z)=\frac{d^{k}}{d z^{k}} E_{\alpha, \beta}(z) .
$$

Considering the specific case for $k=0$ yields

$$
L\left[t^{\beta-1} E_{\alpha, \beta}\left( \pm a t^{\alpha}\right)\right]=\frac{s^{\alpha-\beta}}{s^{\alpha} \mp a} .
$$

And by substituting the Mittag-Leffler function, the following is obtained:

$$
L\left[t^{\beta-1} \sum_{k=0}^{\infty} \frac{\left( \pm a t^{\alpha}\right)^{k}}{\Gamma(\alpha k+\beta)}\right]=\frac{s^{\alpha-\beta}}{s^{\alpha} \mp a} .
$$

Which will be the relation used for calculating the solution in the time domain. 


\section{Dynamic Model of EDLC}

Electric modeling of supercapacitors is an active line of investigation with numerous contributions in the last few years [13-15]. There are not yet totally satisfactory models applicable to any operating mode of the EDLC, allowing the EDLC behavior to be observed and simulated. That is the reason why models based on heuristic techniques are mainly used [14]. Models based on the physics processes occurring in EDLC are also used [15]. Most of the models obtained make use of a considerable number of variables, either of a high number of passive elements or of fractional variables models [16-18]. Either way, testing these devices during long time periods has allowed observing their behavior and their applicability to energy storage systems. Charge and discharge are rapid processes occurring in a short time interval, since high currents are used.

Due to diffusion processes resulting after the charging phase. The evolution of the processes occurring inside the EDLC generates a nonreversing thermal dissipation which makes the assessment of losses in the device possible. This process would occur in both the EDLC charge and discharge phases.

The model of EDLC has been considered as single input (current), single output (voltage) system. The electric model used comes from the model applied to ideal capacitors using integer-order differential equations. Subsequently, a model based on fractional order differential equations is proposed.

3.1. Electric Model of a Conventional Capacitor. The mathematical equation, relating the current and the voltage at the terminals of a real capacitor, can be deduced from an electric circuit made of an ideal capacitor and two parasitic resistors $[9,19]$.

Naming the series resistor as $R_{1}$ and the parallel one as $R_{2}$ and operating the following equation is obtained:

$$
R_{2} \cdot C \frac{d v(t)}{d t}+v(t)=\left(R_{1}+R_{2}\right) \cdot i(t)+R_{1} \cdot R_{2} \cdot C \frac{d i(t)}{d t}
$$

with $v(t)$ being the voltage at the terminals of the EDLC and $i(t)$ its current. The transfer function or impedance in the Laplace domain will be calculated as the quotient between voltage and current

$$
\begin{aligned}
G(s)=\frac{V(s)}{I(s)}= & R_{1}\left(\frac{s}{s+\left(1 /\left(R_{2} \cdot C\right)\right)}\right) \\
& +\frac{R_{1}+R_{2}}{R_{2} \cdot C}\left(\frac{1}{s+\left(1 /\left(R_{2} \cdot C\right)\right)}\right) .
\end{aligned}
$$

Gathering the constants of the transfer function the following is obtained:

$$
G(s)=\frac{V(s)}{I(s)}=\left(k_{1} \frac{s}{s+a}+k_{2} \frac{1}{s+a}\right),
$$

where

$$
k_{1}=R_{1}, \quad k_{2}=\frac{R_{1}+R_{2}}{R_{2} \cdot C}, \quad a=\frac{1}{R_{2} \cdot C} .
$$

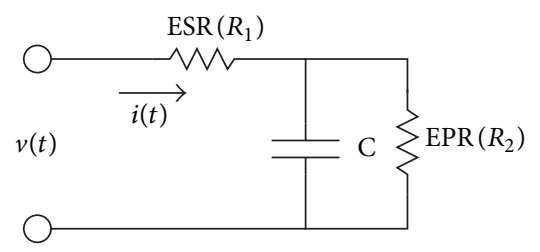

FIGURE 1: Equivalent circuit of a real capacitor.

The differential equation resulting from the transfer function can be solved for simple and complex signals using the existing simulation programs.

3.2. Electric Model of EDLC. The model obtained for real capacitor (12) fits well to the behavior of real capacitors in a wide range of frequencies. However, if applied to an EDLC, the adjustment is unsatisfactory, especially when long time periods are analyzed.

Many papers $[3,10,20]$ exposed the fractional behavior of EDLC. Thus, by changing the ideal capacitor in the model in Figure 1 for a fractional one, the voltage and the current across such fractional capacitor are given by

$$
C \cdot D^{\alpha} v(t)=i(t) \text {. }
$$

Applying this relation to the circuit of Figure 1, it gives a differential equation of the form

$$
{ }_{0} D_{t}^{\alpha} v(t)+a \cdot v(t)=k_{1} \cdot{ }_{0} D_{t}^{\alpha} i(t)+k_{2} \cdot i(t) .
$$

Applying Laplace transform (5) and assuming that the initial conditions are zero, the following is obtained:

$$
G(s)=\frac{V(s)}{I(s)}=\left(k_{1} \frac{s^{\alpha}}{s^{\alpha}+a}+k_{2} \frac{1}{s^{\alpha}+a}\right) .
$$

This transfer function is similar to (14), differing only in the fractional aspect of the capacitor. In this case differential equation (17) is of fractional order, being defined by variable $\alpha$. This equation can be solved for simple and complex signals input, using Mittag-Leffler functions [3, 21].

3.3. Solution to the Fractional Equation of EDLC. In this section the solution to (18) will be deduced in the time domain for a variable input over time.

3.3.1. Solution to a Unitary Step Input. Multiplying transfer function (18) by the unit step input $i(s)=1 / s$, the response of the system to this signal is obtained:

$$
\begin{aligned}
v(s) & =k_{1} \frac{s^{\alpha-1}}{s^{\alpha}+a}+k_{2} \frac{s^{-1}}{s^{\alpha}+a} \\
& \Longrightarrow v(t)=k_{1} L^{-1}\left[\frac{s^{\alpha-1}}{s^{\alpha}+a}\right]+k_{2} L^{-1}\left[\frac{s^{-1}}{s^{\alpha}+a}\right] .
\end{aligned}
$$

Applying relation (11),

$$
v(t)=k_{1} \sum_{j=0}^{\infty} \frac{\left(-a \cdot t^{\alpha}\right)^{j}}{\Gamma(\alpha \cdot j+1)}+k_{2} \cdot t^{\alpha} \cdot \sum_{j=0}^{\infty} \frac{\left(-a \cdot t^{\alpha}\right)^{j}}{\Gamma(\alpha \cdot j+\alpha+1)} .
$$

The response in the time domain to a unit step input is obtained. 
3.3.2. Solution to a Generic Input. The response can be extended to any input. For this purpose, the superposition of steps displaced by the sampling period of the input signal has been used. Thus, for the first input signal, the temporal response matches the response obtained by (20). So, for the first time interval,

$$
\begin{aligned}
& v(t)=i(0) \cdot\left(k_{1} \sum_{j=0}^{\infty} \frac{\left(-a \cdot t^{\alpha}\right)^{j}}{\Gamma(\alpha \cdot j+1)}\right. \\
&\left.+k_{2} \cdot t^{\alpha} \cdot \sum_{j=0}^{\infty} \frac{\left(-a \cdot t^{\alpha}\right)^{j}}{\Gamma(\alpha \cdot j+\alpha+1)}\right), \\
& 0 \leq t \leq T .
\end{aligned}
$$

As the input signal changes in the second period, its response can be obtained by superposing to the previous response a second step, delayed in time, a period $T$, resulting in

$$
\begin{gathered}
v(t)=i(0) \cdot\left(k_{1} \sum_{j=0}^{\infty} \frac{\left(-a \cdot t^{\alpha}\right)^{j}}{\Gamma(\alpha \cdot j+1)}\right. \\
\left.+k_{2} \cdot t^{\alpha} \cdot \sum_{j=0}^{\infty} \frac{\left(-a \cdot t^{\alpha}\right)^{j}}{\Gamma(\alpha \cdot j+\alpha+1)}\right) \\
+(i(T)-i(0)) \cdot\left(k_{1} \sum_{j=0}^{\infty} \frac{\left(-a(t-T)^{\alpha}\right)^{j}}{\Gamma(\alpha \cdot j+1)}\right. \\
\left.+k_{2}(t-T)^{\alpha} \sum_{j=0}^{\infty} \frac{\left(-a(t-T)^{\alpha}\right)^{j}}{\Gamma(\alpha \cdot j+\alpha+1)}\right) \\
T \leq t \leq 2 T .
\end{gathered}
$$

Generalizing (22) for a generic time interval $N$, the following is obtained:

$$
\begin{aligned}
v(t)=\sum_{k=0}^{N}(i(k T)-i((k-1) T)) & \\
& {\left[k_{1} \sum_{j=0}^{\infty} \frac{\left(-a \cdot(t-k T)^{\alpha}\right)^{j}}{\Gamma(\alpha \cdot j+1)}\right.} \\
& \left.+k_{2}(t-k T)^{\alpha} \cdot \sum_{j=0}^{\infty} \frac{\left(-a \cdot(t-k T)^{\alpha}\right)^{j}}{\Gamma(\alpha \cdot j+\alpha+1)}\right], \\
& N T \leq t \leq(N+1) T,
\end{aligned}
$$

where the initial conditions are zero.

\section{Experimental Results, Identification, and Discussion}

4.1. Instruments. The experimental data have been obtained in the laboratory making a test circuit and recording the

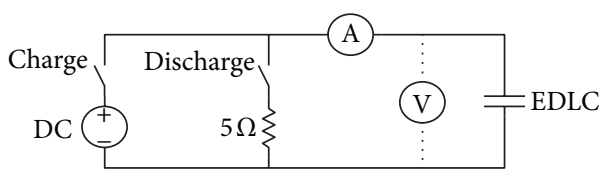

FIgURE 2: Schematic diagram of the experimental circuit.

electrical variables of the EDLC. The analyzed EDLC, manufactured by ELNA, has a capacity of $4.7 \mathrm{~F}$ and a voltage of $2.5 \mathrm{~V}$.

Figure 2 shows the electric circuit for the charge-discharge control. A Crouzet Millenium III PLC has controlled the charge and discharge switches. Voltage and current data have been recorded through a NI USB-6009 data logger by National Instruments.

4.2. Experimental Results. Several tests have been performed to the EDLC in order to monitor voltage and current signals. Because EDLC have memory effect, just before each test they have been short-circuited for 24 hours to ensure zero initial conditions.

The tests were performed as follows. In a first phase lasting a few minutes, was charged at a constant current between $20 \mathrm{~mA}$ and $0.9 \mathrm{~A}$ until the voltage reached $2.4 \mathrm{~V}$; in the second phase, the current is cut off and left to rest for 8 hours to allow the voltage stabilization, and finally in the third phase is discharged through a resistance of $5 \mathrm{ohm}$ until it was fully discharged.

Although the sampling period of voltage and current has been recorded at $0.1 \mathrm{~s}$; sfor identification purposes it has been considered a $5 \mathrm{~s}$ sampling time. Figure 3 shows the experimental data obtained. The evolution of voltage in the EDLC throughout the process can be appreciated in Figure 3(a), while the current supplied at Figure 3(b). They are observed perfectly the three stages described above.

4.3. Identification. The coefficients for the proposed model have been defined according to transfer function (18) and implemented using (23).

For identification the MATLAB simulation software was used. The function created has as input parameters $\alpha, a, k_{1}$, and $k_{2}$. Using (23) the voltage response is generated taking all the current samples as input. The voltage is compared with the experimental data through standard deviation (24), generating the output of the function. Using this function in the command fminsearch of MATLAB, sought parameters are obtained. For implementing (23), the terms $j$ of the sum may be truncated without causing significant errors.

The index chosen in this paper is the standard deviation defined as

$$
\sigma_{d}=\sqrt{\frac{\sum_{i=1}^{N}\left(y_{i, \exp }-y_{i, \mathrm{cal}}\right)^{2}}{N-1}},
$$

where $N$ is the number of samples and subindexes which represent the values obtained experimentally and calculated according to the model. 


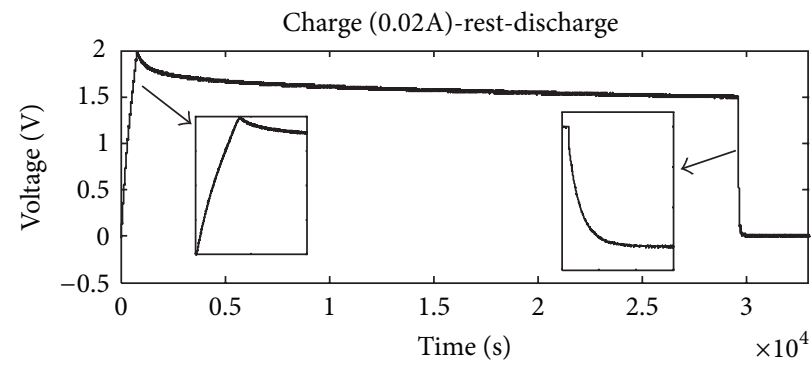

(a)

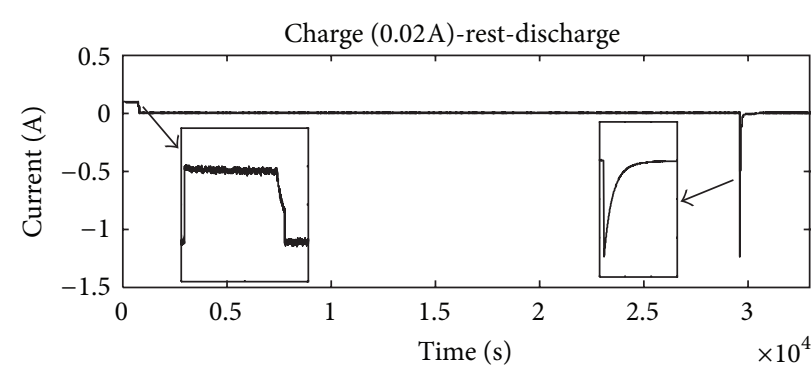

(b)

Figure 3: Charge-rest-discharge at $20 \mathrm{~mA}$.

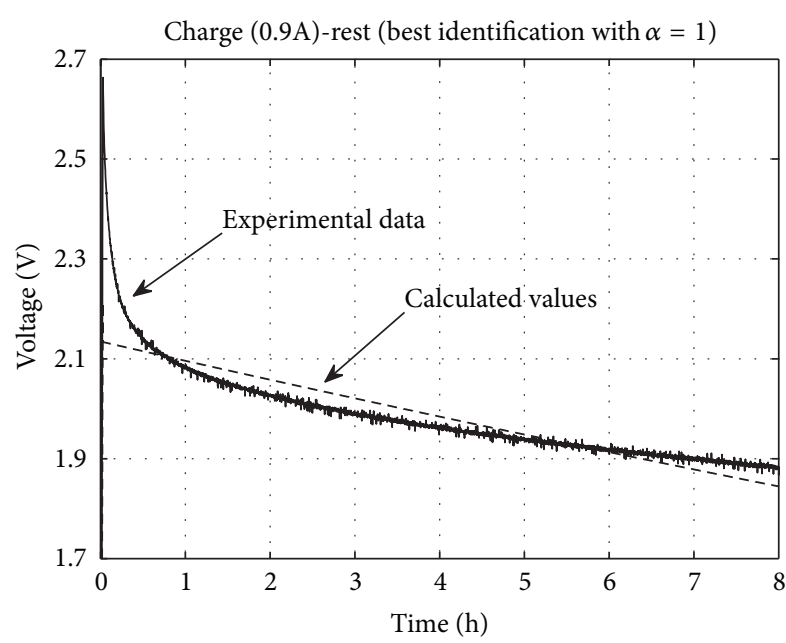

FIGURE 4: Fitting in the resting phase using integer derivatives.

TABLE 1: Charge + rest identification parameters.

\begin{tabular}{lcccccc}
\hline Test & $I(\mathrm{~A})$ & $\alpha$ & $a$ & $k_{1}$ & $k_{2}$ & $\sigma_{d}$ \\
\hline 1 & $0.02 \mathrm{~A}$ & 0.941 & $5.974 E-08$ & 0.239 & 0.202 & $2.079 E-02$ \\
2 & $0.1 \mathrm{~A}$ & 0.952 & $8.671 E-08$ & 0.261 & 0.187 & $1.297 E-02$ \\
3 & $0.5 \mathrm{~A}$ & 0.950 & $1.02 E-07$ & 0.267 & 0.170 & $1.354 E-02$ \\
4 & $0.5 \mathrm{~A}$ & 0.950 & $1.020 E-07$ & 0.250 & 0.169 & $1.338 E-02$ \\
5 & $0.9 \mathrm{~A}$ & 0.953 & $9.917 E-08$ & 0.228 & 0.164 & $1.096 E-02$ \\
\hline
\end{tabular}

The EDLC identification has been carried out calculating the parameters $\alpha, a, k_{1}$, and $k_{2}$ in (18) and has consisted of two parts: first, from the beginning until just before discharged and second, from the discharge onwards. This is because the internal processes in the EDLC are different $[22,23]$.

The data obtained for the charge and rest phases are shown in Table 1.

Considering relation (15) between the parameters calculated with the resistors and the capacity in Figure 1, the parameters obtained for the charge are in Table 2.

In order to compare the adjustment with traditional model (14) in which the fractional index is an integer, Table 3 and Figure 4 show the best fit to the data of test number 5 setting $\alpha=1$.
TABLE 2: Charge + rest electrical parameters.

\begin{tabular}{lccccc}
\hline Test & $I(\mathrm{~A})$ & $\alpha$ & $C$ & $R_{1}$ & $R_{2}$ \\
\hline 1 & $0.02 \mathrm{~A}$ & 0.941 & 4.95 & 0.239 & $3.382 E+06$ \\
2 & $0.1 \mathrm{~A}$ & 0.952 & 5.35 & 0.261 & $2.155 E+06$ \\
3 & $0.5 \mathrm{~A}$ & 0.950 & 5.89 & 0.267 & $1.660 E+06$ \\
4 & $0.5 \mathrm{~A}$ & 0.950 & 5.91 & 0.250 & $1.660 E+06$ \\
5 & $0.9 \mathrm{~A}$ & 0.953 & 6.08 & 0.228 & $1.658 E+06$ \\
\hline
\end{tabular}

TABLE 3: Parameters calculated for test number 5 setting $\alpha=1$.

\begin{tabular}{lcccc}
\hline Test & $\alpha$ & $C$ & $R_{1}$ & $R_{2}$ \\
\hline 1 & 1.000 & 9.00 & 1.262 & $2.191 E+04$ \\
\hline
\end{tabular}

TABLE 4: Discharge identification parameters.

\begin{tabular}{lccccc}
\hline Test & $\alpha$ & $a$ & $k_{1}$ & $k_{2}$ & $\sigma_{d}$ \\
\hline 1 & 0.975 & $9.600 E-08$ & 0.235 & 0.187 & $1.391 E-02$ \\
2 & 0.981 & $9.282 E-08$ & 0.244 & 0.185 & $1.281 E-02$ \\
3 & 0.978 & $8.427 E-08$ & 0.267 & 0.192 & $2.068 E-02$ \\
4 & 0.976 & $1.556 E-07$ & 0.251 & 0.202 & $2.338 E-02$ \\
5 & 0.982 & $1.032 E-07$ & 0.240 & 0.171 & $1.285 E-02$ \\
\hline
\end{tabular}

TABLE 5: Discharge electrical parameters.

\begin{tabular}{lcccc}
\hline Test & $\alpha$ & $C$ & $R_{1}$ & $R_{2}$ \\
\hline 1 & 0.975 & 5.34 & 0.235 & $1.950 E+06$ \\
2 & 0.981 & 5.40 & 0.244 & $1.996 E+06$ \\
3 & 0.978 & 5.21 & 0.267 & $2.278 E+06$ \\
4 & 0.976 & 4.95 & 0.251 & $1.297 E+06$ \\
5 & 0.982 & 5.85 & 0.240 & $1.656 E+06$ \\
\hline
\end{tabular}

Operating in a similar way, the parameters for the discharge through the $5 \Omega$ resistor are in Table 4 .

Of which the following electric parameters are obtained in Table 5.

The following figures show graphically the fit of the proposed model with experimental data. Figure 5 shows that the model has a good precision all over the time range tested. Figures 6 and 8 display the model and the experimental data in the charging and discharging phases. Figures 4 and 7 show the fit when using transfer functions with integer and 


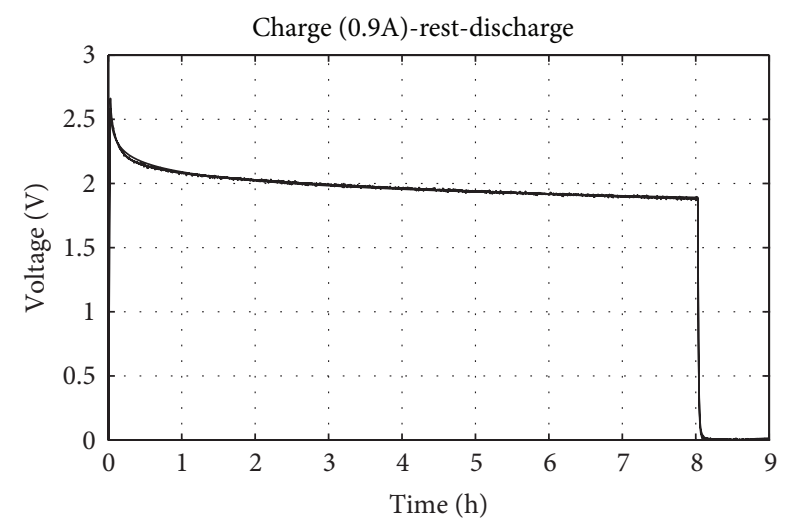

FIGURE 5: Experimental data of test number 5 versus proposed model.

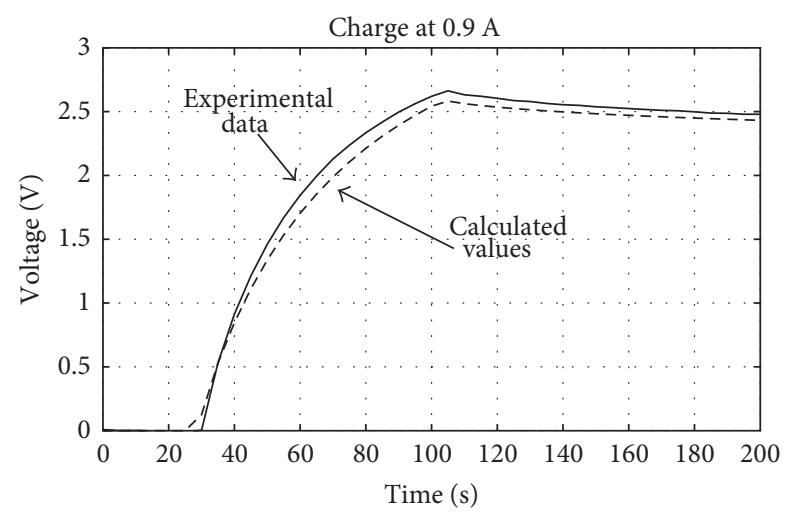

FIGURE 6: Detail of fitting in charging phase.

fractional indices, showing that the fit with the traditional model gives unsatisfactory results. In summary, it can be observed that the voltage deduced through the fractional model adjusts very close to the real data even in long time periods.

4.4. Discussion. The self-discharge adjustment of EDLC after charging and after long time resting phases is a complex phenomenon which is not easily applicable to modeling through integer-order transfer functions. In this work the EDLC has been modeled making use of the fractional derivative. Taking the electric circuit in Figure 1 as starting point and assuming that the capacitor has a fractional index $\alpha$, fractional transfer function (18) has been deduced. This function has been solved in the time domain using Mittag-Leffler functions and implemented in a simulation program through (23).

First of all, considering Figure 5, it can be observed that the proposed transfer function adjusts well to the real data, modeling satisfactorily the self-discharge phenomenon.

Unlike the traditional capacitor model, which is defined by the value of two resistors and the capacity, the proposed model adds a new parameter, that is, the fractional index $\alpha$ of the fractional capacitor. This new parameter models to a greater extent the self-discharging phenomenon.

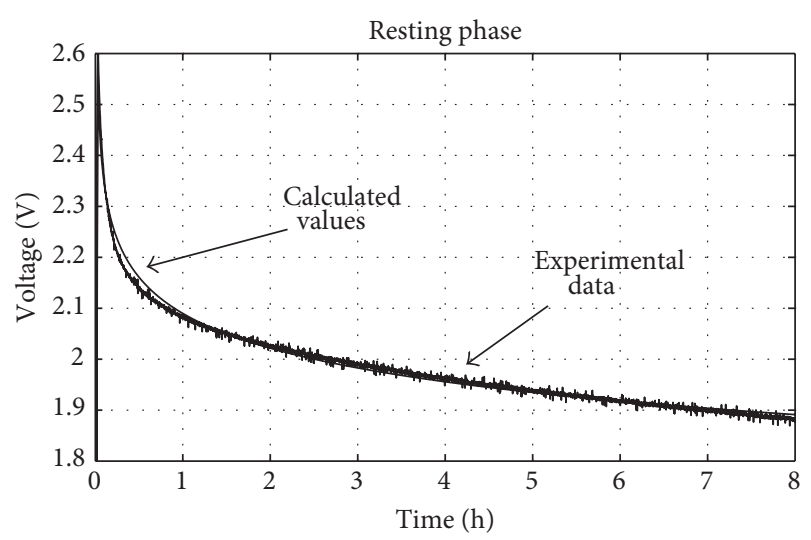

FIGURE 7: Fitting in the resting phase using the proposed model.

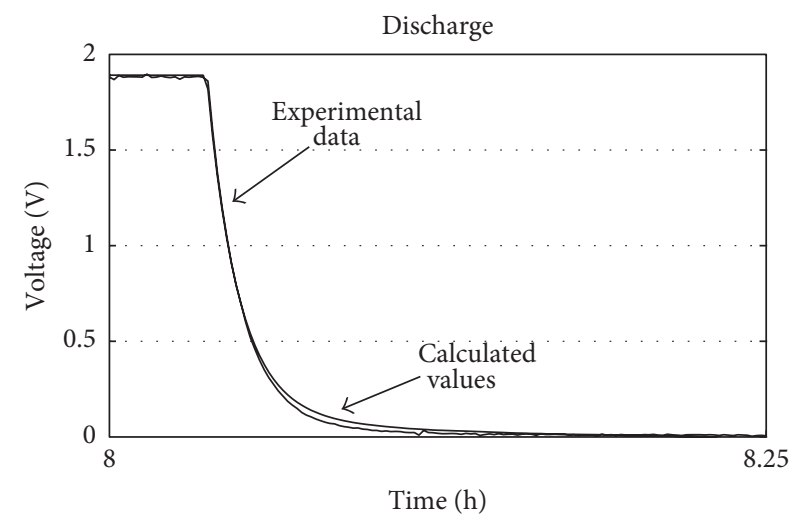

FIGURE 8: Fitting in the discharging phase.

Although constants in Tables 1 and 4 have been taken for the identification of equations, constants in Tables 2 and 5 will provide the data related to the value of the electric elements.

In all cases the adjustment has been very precise, with the typical deviation being less than 0.03 .

The constancy of $R_{1}, R_{2}$, and $C$ values for both charge and discharge is especially noteworthy.

At last, the most significant difference in terms of charge and discharge is given by the value of the fractional index $\alpha$, which is around 0.95 in the charge and around 0.98 in the discharge.

\section{Conclusions}

This work has consisted of EDLC modeling by implementing a simple fractional model allowing a very satisfactory response for long time periods. Traditional models based on transfer functions with integer coefficients yield good results in those cases when there is no self-discharge phenomenon. Applying fractional mathematics implies an additional parameter to the already necessary parameters in order to define the basic model shown in Figure 1, which is the fractional index $\alpha$. According to the tests this index has different values for the charge and the discharge, with those 
values being apparently independent of the EDLC charging current.

Thanks to the proposed transfer function and the MittagLeffler functions, it is possible to estimate the voltage at the EDLC terminals after a long time, taking into account the charging current and the way it has been charged.

\section{References}

[1] A. Burke, "Ultracapacitors: why, how, and where is the technology," Journal of Power Sources, vol. 91, no. 1, pp. 37-50, 2000.

[2] B. Conway, Electrochemical Supercapacitors. Scientific Fundamentals and Technological Applications, Kluwer Academic, New York, NY, USA, 1999.

[3] I. Podlubny, Fractional Differential Equations, vol. 198 of Mathematics in Science and Engineering, Academic Press, San Diego, Calif, USA, 1999

[4] K. B. Oldham and J. Spanier, The Fractional Calculus, vol. 1047, Academic Press, New York, NY, USA, 1974.

[5] K. S. Miller and B. Ross, An Introduction to the Fractional Calculus and Fractional Differential Equations, John Wiley \& Sons, New York, NY, USA, 1993.

[6] A. Oustaloup, A. Ballouk, and B. Bansard, "Partial differential equations and non integer derivation," in Proceedings of the International Conference on Systems, Man and Cybernetics, pp. 166-173, October 1993.

[7] B. Mbodje and G. Montseny, "Boundary fractional derivative control of the wave equation," IEEE Transactions on Automatic Control, vol. 40, no. 2, pp. 378-382, 1995.

[8] G. Montseny, J. Audounet, and B. Mbodje, "Optimal model of fractional integrators and application to systems with fading memory," in Proceedings of the International Conference on Systems, Man and Cybernetics, pp. 65-70, October 1993.

[9] S. Westerlund and L. Ekstam, "Capacitor theory," IEEE Transactions on Dielectrics and Electrical Insulation, vol. 1, no. 5, pp. 826-839, 1994.

[10] N. Bano and R. A. Hashmi, "Electrical studies of leaves over wide frequency range," IEEE Transactions on Dielectrics and Electrical Insulation, vol. 3, no. 2, pp. 229-232, 1996.

[11] G. E. Carlson and C. A. Halijak, "Simulation of the fractional derivate operator and the fractional integral operator $\sqrt{s}$," in Proceedings of the Central State Simulation Council Meeting, vol. 45, no. 7, Kansas State University, May 1961.

[12] T. C. Haba, M. Martos, G. Ablart, and P. Bidan, "Composants électroniques à impédance fractionnaire," in Proceedings of the ESAIM conferece on Fractional Differential Systems: Models, Methods and Applications, vol. 5, pp. 99-109, 1998.

[13] J. J. Quintana, A. Ramos, and I. Nuez, "Identificaction of the fractional impedance of ultracapacitors," in Proceedings of the IFAC Workshop (FDA'06), Oporto, Portugal, 2006.

[14] L. Zubieta and R. Bonert, "Characterization of double-layer capacitors for power electronics applications," IEEE Transactions on Industry Applications, vol. 36, no. 1, pp. 199-205, 2000.

[15] N. Bertrand, J. Sabatier, O. Briat, and J.-M. Vinassa, "Embedded fractional nonlinear supercapacitor model and its parametric estimation method," IEEE Transactions on Industrial Electronics, vol. 57, no. 12, pp. 3991-4000, 2010.

[16] R. Martín, J. J. Quintana, A. Ramos, and I. de la Nuez, "Modeling of electrochemical double layer capacitors by means of fractional impedance," Journal of Computational and Nonlinear
Dynamics, vol. 3, no. 2, Article ID 021303, pp. 21303-21400, 2008.

[17] V. Musolino, L. Piegari, and E. Tironi, "New full-frequencyrange supercapacitor model with easy identification procedure," IEEE Transactions on Industrial Electronics, vol. 60, no. 1, pp. 112-120, 2013.

[18] Y. Wang, T. Hartley, C. Lorenzo, and J. Adams, Modeling Ultracapacitors as Fractional-Order Systems, New Trends in Nanotechnology and Fractional Calculus Applications, 2010.

[19] R. L. Spyker, "Classical equivalent circuit parameters for a double-layer capacitor," IEEE Transactions on Aerospace and Electronic Systems, vol. 36, no. 3, pp. 829-836, 2000.

[20] A. Dzieliński, G. Sarwas, and D. Sierociuk, "Time domain validation of ultracapacitor fractional order model," in Proceedings of the 49th IEEE Conference on Decision and Control (CDC '10), pp. 3730-3735, December 2010.

[21] T. J. Freeborn, B. Maundy, and A. S. Elwakil, "Measurement of supercapacitor fractional-order model parameters from voltage-excited step response," IEEE Journal on Emerging and Selected Topics in Circuits and Systems, vol. 3, no. 3, pp. 416-424, 2013.

[22] R. de Levie, "On porous electrodes in electrolyte solutions. I. Capacitance effects," Electrochimica Acta, vol. 8, no. 10, pp. 751780, 1963.

[23] M. Kaus, J. Kowal, and D. U. Sauer, "Modelling the effects of charge redistribution during self-discharge of supercapacitors," Electrochimica Acta, vol. 55, no. 25, pp. 7516-7523, 2010. 


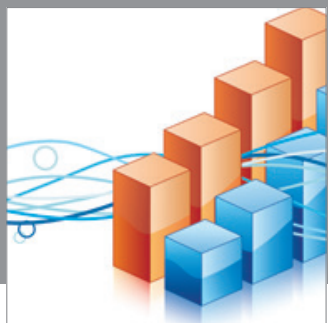

Advances in

Operations Research

mansans

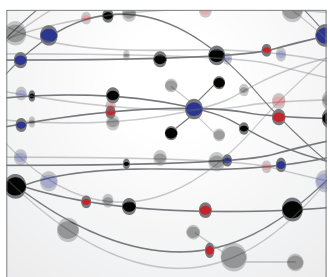

The Scientific World Journal

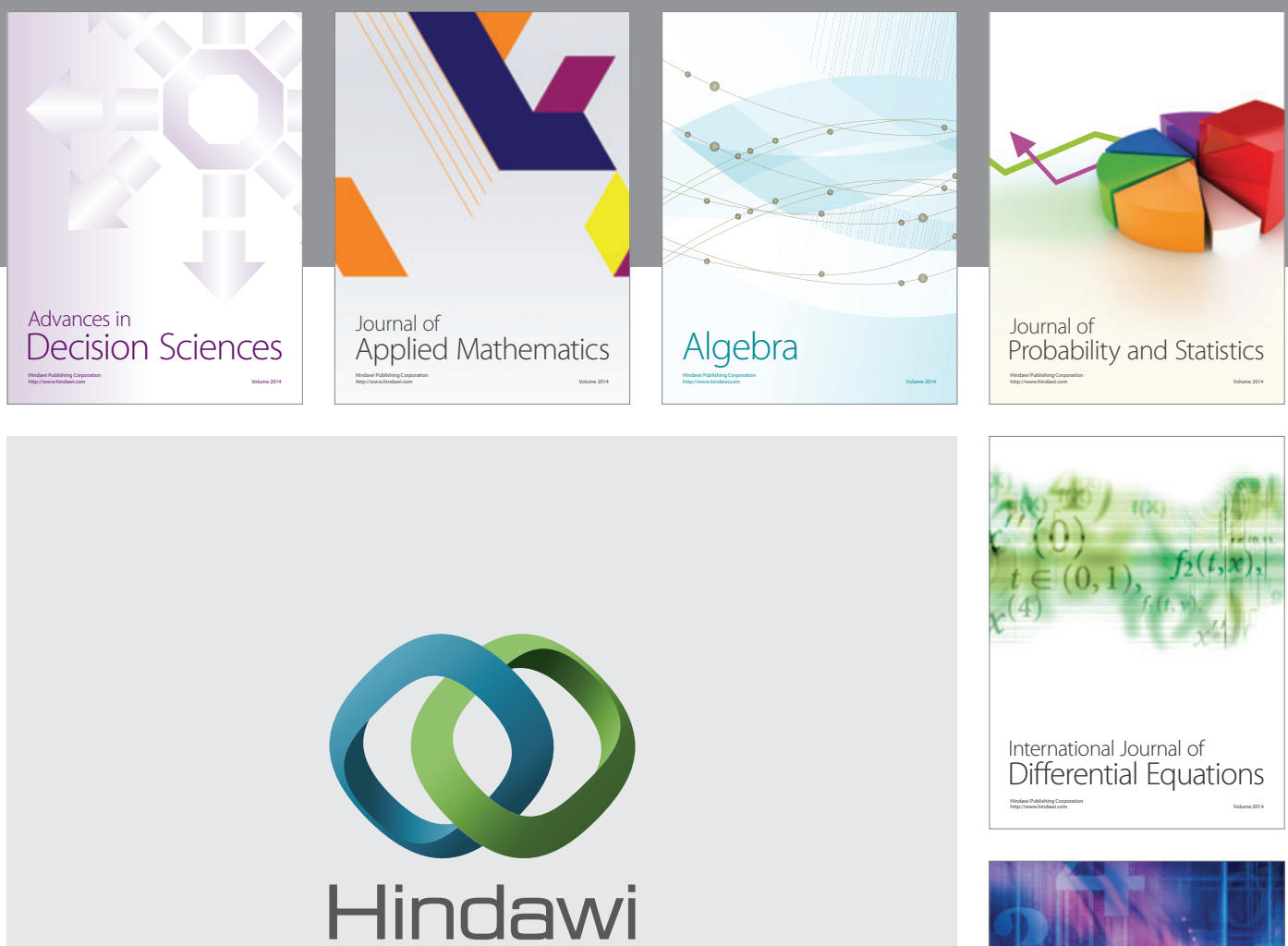

Submit your manuscripts at http://www.hindawi.com
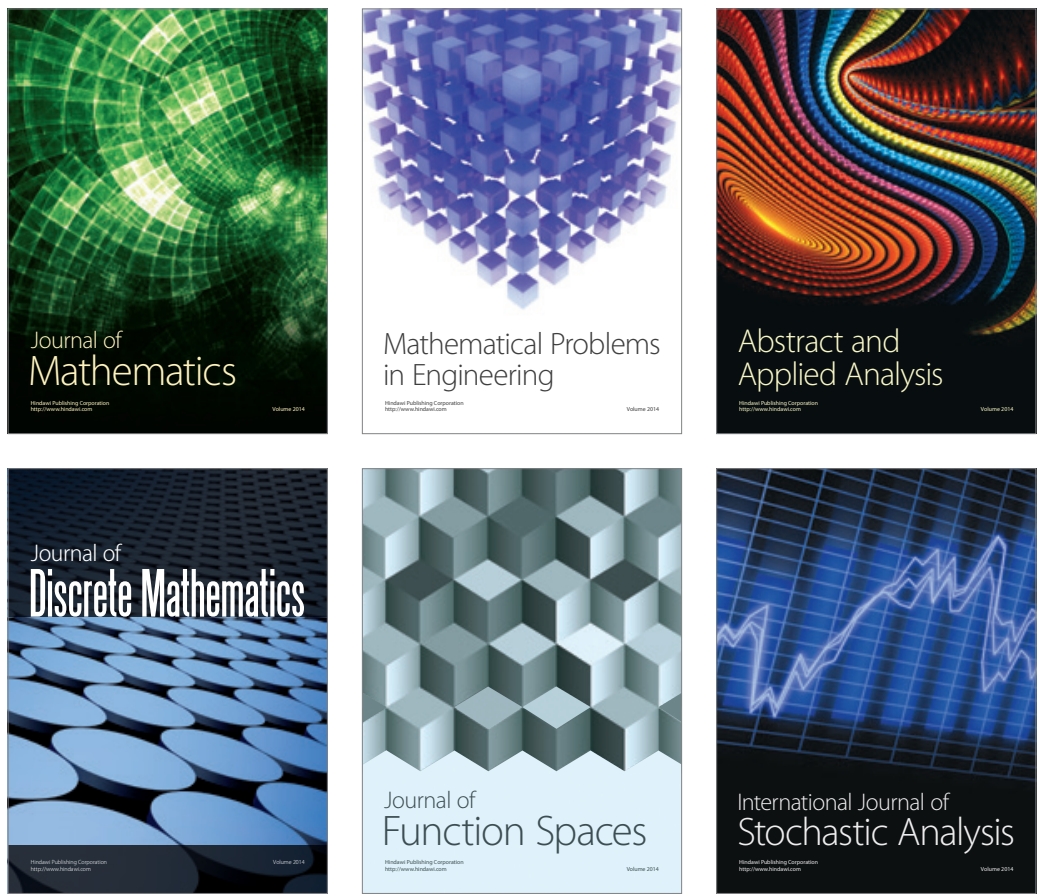

Journal of

Function Spaces

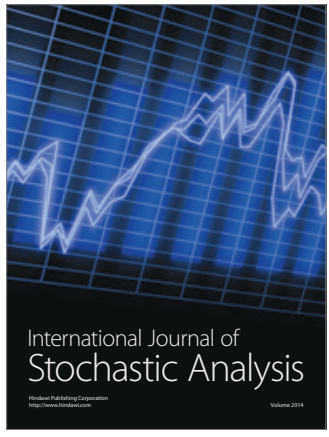

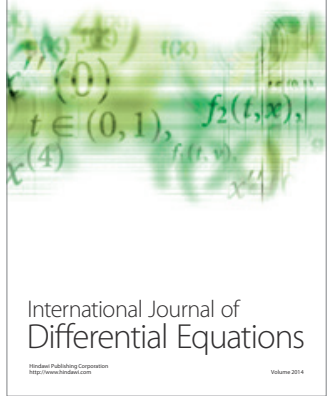
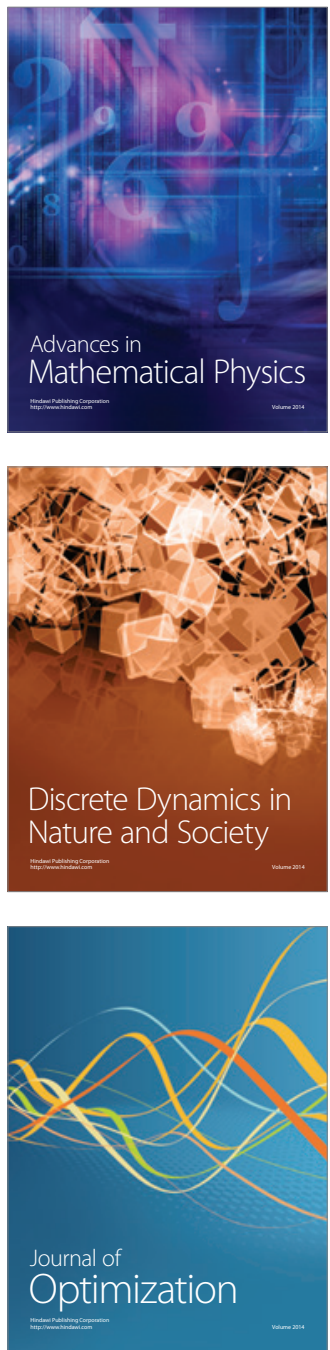